\title{
RAMAN MICROSPECTROSCOPY OF SOME HIGH EXPLOSIVES, - REVISITED
}

\author{
Edgar S. Etz, Sonya V. Roberson, and Greg Gillen \\ Chemical Science and Technology Laboratory, NIST, Gaithersburg, MD 20899-8371, U.S.A.
}

Following the events of September 11, 2001, a number of analytical methodologies are being re-examined for their utility and effectiveness in the war on terror. As a result, the detection and identification of trace explosives has become a matter of some urgency, with airport security a top priority. Various technologies have been applied to the trace detection of explosives ${ }^{(1,2)}$, such as ion mobility spectrometry (IMS) and electron capture detection. These have sensitivities as low as parts-per-trillion (ppt) which make them potentially viable tools in the screening for explosives. Concurrently, other methodologies are being reexamined, such as Raman spectroscopy, as fieldable instrumentation has been developed for sampling and analysis. Raman microanalysis has a proven track record for dealing with microparticulate explosives. ${ }^{(3-5)}$ These studies have shown that modern Raman instruments provide for sensitive detection of particulate explosives, for single particles down to $\sim 2 \mu \mathrm{m}$ in size.

In a new program focused at examining the analytical potential of time-of-flight (TOF) secondary ion mass spectrometry (SIMS) for the characterization of high explosives (HEs), we are re-visiting the micro-Raman methods not only for their intrinsic merits as an analytical tool, but also to elucidate some aspects of the TOFSIMS approach. The Raman spectrum is a fingerprint of molecular (vibrational) structure and potentially can answer questions about the fragmentation of HEs by thermal decomposition reactions that result from the bombardment of the explosive by the polyatomic primary ion beam used in TOF-SIMS. We have acquired the micro-Raman spectra of a set of high explosives samples, studied under ambient conditions, using two types of Raman microprobes. Both are commercial instruments. One is a dispersive/scanning system utilizing $785 \mathrm{~nm}$ (Ti:sapphire) excitation, the second a Fourier-transform (FT) system with $1064 \mathrm{~nm}$ (Nd:YAG) laser excitation. Among the samples analyzed are several compositions of common plastic explosives, such as Semtex-H and C-4, which are binary composites consisting of RDX (cyclo-1,3,5-trimethylene-2,4,6-trinitramine) and PETN (pentaerythritol tetranitrate), plus several additives. Their micro-Raman spectra are shown in Figs. 1-3, generally taken from single, microscopic particles or crystallites. The neat (i.e., pure) HEs (RDX and PETN) show no background luminescence at either 785 or $1064 \mathrm{~nm}$. The composites (Semtex-H and C-4), however, show more luminescence at $785 \mathrm{~nm}$ than they do under $1064 \mathrm{~nm}$ excitation. Yet both excitation schemes provide useful and informative spectra. Clearly, in the case of the two plastic explosives, the component HEs are readily detected and identified. Further, micro-Raman offers a unique opportunity to analyze individual microparticles of explosives in situ, on collection surfaces. We are now analyzing particulate deposits on typical sampling cloths used by the FAA (Federal Aviation Administration) for routine screening at airport portals and for performance evaluation tests of trace explosives detection systems.

These studies are now being extended to sample temperatures above ambient, approaching the sublimation or melting regime of both RDX and PETN, to study the vaporization and thermal decomposition of the explosive involving the breakage of covalent bonds. This has relevance to the SIMS measurements based on HE fragmentation to form the negative secondary ions that are observed in the mass spectrum, and which determine secondary ion yields. The results are expected to furnish information relative to the ion mobility spectra that are produced by IMS involving atmospheric pressure chemical ionization reactions. We will discuss the Raman spectra obtained from these HE decomposition studies with reference to the SIMS and IMS ionization mechanisms for these explosives.

[1] Susan F. Hallowell, Talanta 54 (2001) 447-458.

[2] R.G. Ewing, D.A. Atkinson, G.A. Eiceman, and G.J. Ewing, Talanta 54 (2001) 515-529.

[3] I.R. Lewis, N.W. Daniel, Jr., N.C. Chaffin, P.R. Griffiths, and M.W. Tungol, Spectrochim. Acta Part A 51 (1995) 1985-2000.

[4] C. Cheng, T.E. Kirkbride, D.N. Batchelder, R.J. Lacey, and T.G. Sheldon, J.Forensic Sci. 40 (1995) 31-37.

[5] N.F. Fell et al., J. Raman Spectrosc. 27 (1996) 97-104. 

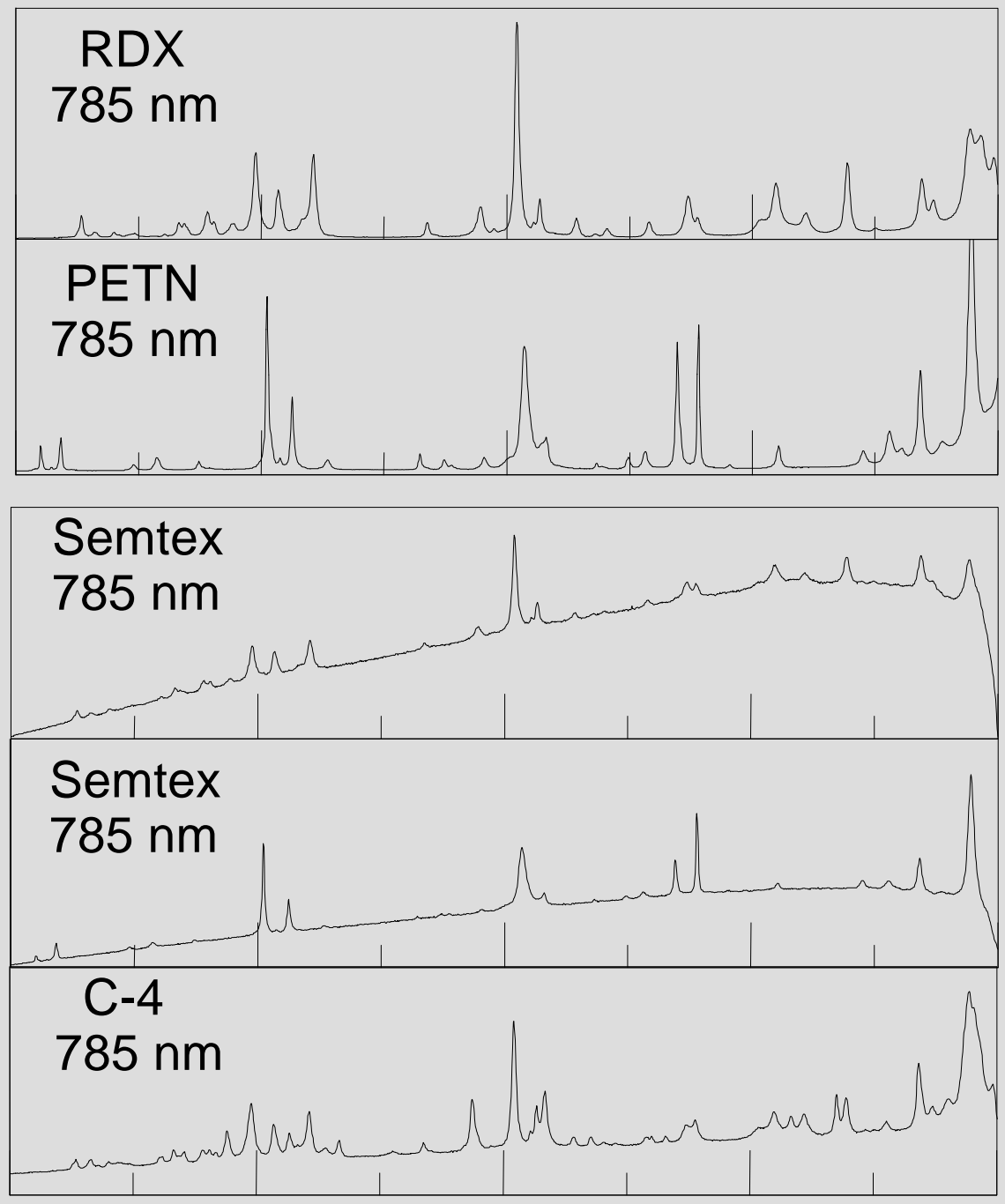

FIG. 1, top: Representative microRaman spectra, excited at $785 \mathrm{~nm}$, of the neat explosives RDX and PETN. In each case, the spectrum is that of a single particle $\sim 10 \mu \mathrm{m}$ in size. At this laser wavelength, the spectra exhibit no sample luminescence. The spectra are polarization scrambled.

FIG.2, middle: The micro-Raman spectra, excited at $785 \mathrm{~nm}$, of two arbitrary particles of Semtex-H (composition: 35:35 wt\% RDX: PETN in $70 \mathrm{wt} \%$ total explosive; $\sim 8 \mathrm{wt} \%$ oil, plus plasticiser, antioxidant, and dye (total $22 \mathrm{wt} \%$ )), and of a particle of C-4 explosive (composition: $91 \mathrm{wt} \% \mathrm{RDX}$ plus $\sim 9 \mathrm{wt} \%$ plasticiser). The spectra of both plastic explosives show varying levels of luminescence.

FIG.3, bottom: FT-Raman spectra, excited at $1064 \mathrm{~nm}$, of Semtex-H, sampled in the macro-mode (beam spot $\sim 200 \mu \mathrm{m}$ ) and probed in the micro-mode (laser spot size $\sim 4 \mu \mathrm{m}$ ) The macro- spectrum is the averaged spectrum from a particle aggregate of the explosive.

1700

1300

900

500

100

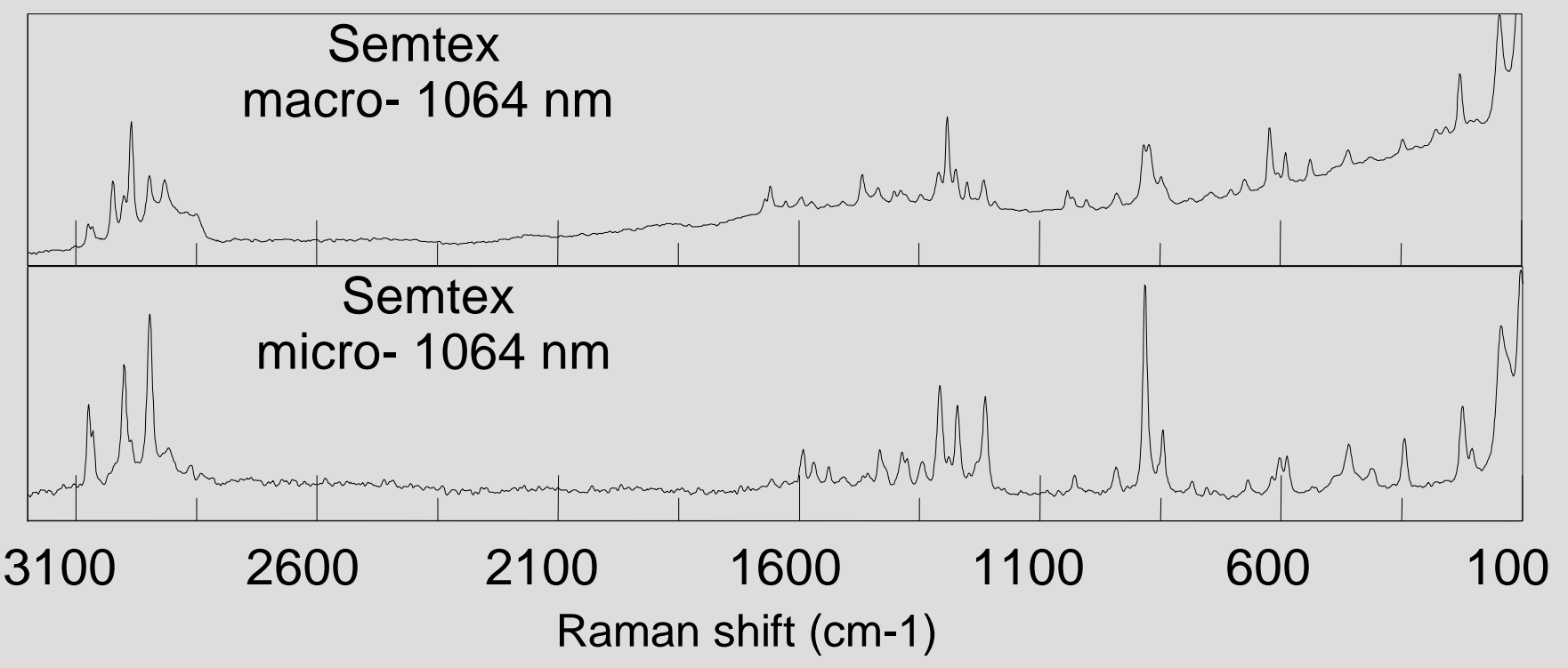

